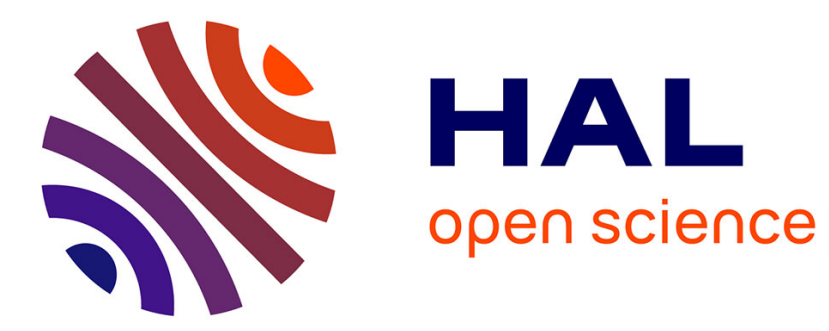

\title{
Lossless and Lossy Minimal Redundancy Pyramidal Decomposition for Scalable Image Compression Technique
}

\author{
Marie Babel, Olivier Déforges, Joseph Ronsin
}

\section{- To cite this version:}

Marie Babel, Olivier Déforges, Joseph Ronsin. Lossless and Lossy Minimal Redundancy Pyramidal Decomposition for Scalable Image Compression Technique. Apr 2003, pp.249-252. hal-00132654

\section{HAL Id: hal-00132654 \\ https://hal.science/hal-00132654}

Submitted on 22 Feb 2007

HAL is a multi-disciplinary open access archive for the deposit and dissemination of scientific research documents, whether they are published or not. The documents may come from teaching and research institutions in France or abroad, or from public or private research centers.
L'archive ouverte pluridisciplinaire HAL, est destinée au dépôt et à la diffusion de documents scientifiques de niveau recherche, publiés ou non, émanant des établissements d'enseignement et de recherche français ou étrangers, des laboratoires publics ou privés. 


\title{
LOSSLESS AND LOSSY MINIMAL REDUNDANCY PYRAMIDAL DECOMPOSITION FOR SCALABLE IMAGE COMPRESSION TECHNIQUE
}

\author{
Marie Babel, Olivier Déforges \\ UMR CNRS 6164 IETR Groupe Image, INSA de Rennes \\ 20, av. des Buttes de Cosmes, 35043 RENNES, FRANCE \\ Contact : \{mbabel,odeforges\}@insa-rennes.fr
}

\begin{abstract}
We present a new scalable compression technique dealing simultaneously with both lossy and lossless image coding. An original DPCM scheme with refined context is introduced through a pyramidal decomposition adapted to the LAR (Locally Adaptive Resolution) method, which becomes by this way fully progressive. An implicit context modeling of the prediction errors, due to the low resolution image representation including variable block size structure, is then exploited to the for lossless compression purpose.
\end{abstract}

\section{INTRODUCTION}

Recently, many techniques have been developed in the field of lossless compression of greyscale images. The state-ofthe art mentions the use of predictors or reversible transforms. Moreover, standards using predictions schemes are mostly non-scalable, such as the well-known CALIC method [1]. Nevertheless, S+P [2] algorithm proposes a multiresolution decomposition of images, combining S-Transform and prediction.

Besides, TMW [3] introduced the interesting principle of a description for an image in two parts. By this way, images are considered to be the superposition of the global information and the texture (local information). This concept allows us to provide either a lossy (first part of the message) or a lossless compression (second part, corresponding to the error image).

The LAR (Locally Adaptive Resolution) [4] based on a variable block-size decomposition and leading to an efficient lossy image compression technique is shown in the first section. The purpose of this paper is to present a new lossless and lossy image coding, given by the improvement of the LAR coder, that combines both prediction (DPCM scheme) and scalability. Section 3 defines a minimal-redundancy pyramidal decomposition that allows a progressive reconstruction at the decoder. The last part of this paper presents the lossless system of coding, that takes advantage of the implicit context modeling property of our decomposition for entropy encoding.

\section{LAR METHOD FOR GREYSCALE IMAGES}

The LAR compression method - designed first for lossy greyscale images coding - is a two-layer codec : a spatial codec and a complementary spectral one. The spatial coder provides a low bit rate compressed image whereas the spectral one encodes the texture. The LAR method was also extended for region-based color image coding. Furthermore the quality of the low resolution LAR image has been evaluated and recognized to be better than Jpeg-2000 [4].

\subsection{Spatial coder}

The basic idea is that the local resolution, i.e. the pixel size, can depend on the local activity. This leads to construct a variable image resolution, determined by means of a quadtree data structure. The final size of pixels (from $16 \times 16$ to $2 \times 2$ ) is defined thanks to a local gradient estimation : each block is reconstructed by its average luminance. Through this decomposition, the pixel size gives implicitly the nature of the blocks : indeed, small ones are located on contours whereas large ones are situated on smooth areas (Fig. 1). Consequently, a coarse edge-driven segmentation map is automatically available at both the coder and the decoder.

This image content information controls a psychovisual quantization of the luminance : large squares require a fine quantization (in uniform areas, human eyes are strongly sensitive to brightness variations) and small ones can support a coarse quantization (low sensitivity). Perceptible blocks artifacts in homogenous areas are easily removed by a simple but efficient post-processing based on an adaptive linear interpolation.

To sum up, this coder has mainly two distinctive characteristics : on one hand, we have a very fast and efficient technique for high compression ratio, and on the other hand, the method simplifies the image source by removing the local texture while preserving the objects boundaries. 


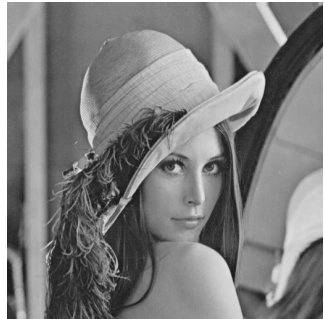

(a)

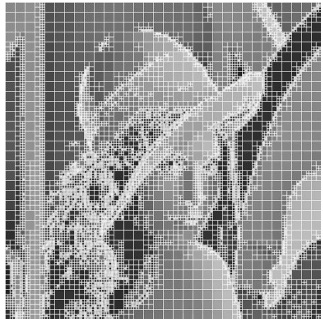

(b)
Fig. 1. (a) Original image. (b) Variable block sizes representation (subsampled grid : $0.032 \mathrm{bpp}$ ).

\subsection{Spectral coder}

In order to obtain higher image quality, the texture (whole error image) can be encoded by the spectral coder that uses a DCT adaptive block-size approach [5]. Both the size and the DC components are here provided by the first coder. The use of adapted square size allows a content-based scalable encoding scheme : for example, edge enhancement can be made only by transmitting the AC coefficients of small blocks.

\section{LOSSY PYRAMIDAL PREDICTIVE DECOMPOSITION}

In this section we present the new progressive approach, that combines prediction (DPCM) and pyramidal decomposition. This leads to construct a scalable LAR spatial coder.

\subsection{Prediction algorithm}

The actual spatial LAR coder encodes in one pass (raster scan) the intensity block values by means of the Gradient Adjusted Predictor (GAP) [1]. The new scalable DPCM scheme is based on the predictor described in [6] by $\mathrm{Wu}$. For full resolution image, errors are coded by means of three interlaced sampling of the original image. By this way, we tend to obtain a spatial configuration of $360^{\circ}$ type context surrounding a given pixel.

The general principle is as follows (Fig. 2) : the first pass encodes a uniform subsampled image formed by the average of two diagonally adjacent pixels through a classical DPCM system. Then the second pass provides the values of the two pixels used to compute the previous image. At this stage, the prediction $360^{\circ}$ type context consists in the already known values of the current pass and the diagonal means coded by the first pass. Finally, the third pass encodes the remaining half of the original image. Once again, thanks to the reconstructed pixels, a completely spatially enclosing and adjacent context is available to predict the modeled pixel.

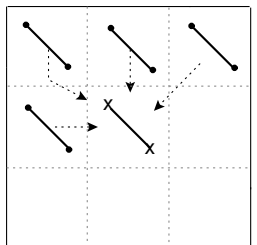

(a) 1st pass

- Pixel already coded - Values used for prediction

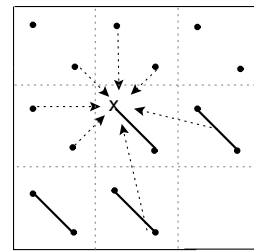

(b) 2nd pass

\subsection{Minimal redundancy pyramidal decomposition}

We propose to extend the previous full resolution scanning scheme to get a pyramidal representation of the image.

Bottom-Up building. Four blocks $\frac{N}{2} \times \frac{N}{2}$ are gathered into one block $N \times N$ valued by the average of the two blocks of the first diagonal (Fig. 3). By this way, the value of a $N \times N$ is equal to the average over the whole diagonal in the full resolution image. The highest level (level 4) of the pyramid corresponds to the $16 \times 16$ blocks (largest block size in the LAR method).

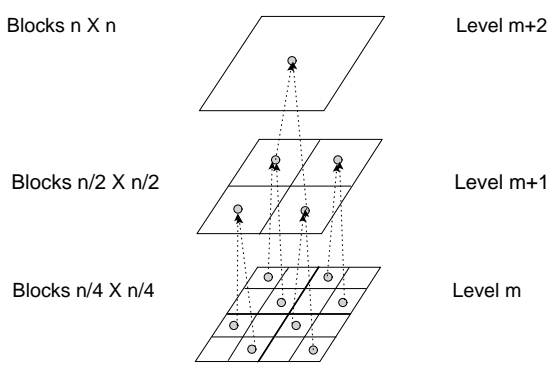

Fig. 3. Bottom-up building.

Top-Down decomposition. Let the original image be of width $W$ and height $H$, the first stage of our pyramid concerns the image of size $W / 16 \times H / 16$ (level 4). The first pass of the Wu algorithm (simple causal DPCM coding of the block values) is applied. Then, each square is split until its maximal size is reached. For a given level (block size) of the pyramid, the blocks of smaller or equal size are successively processed by passes 2 and 3 . The values of higher size blocks are copied out in order to refine the context (Fig. 4) .

By this way, taking into account the variable resolution LAR image, a progressive content-based method is developed : if a restricted context is sufficient for the large blocks, a more accurate and informative context exists for the small blocks (objects boundaries). The main property of this method is that the redundancy is minimal. Indeed, for a group of four blocks obtained by splitting a block from the above level, the diagonal mean is already known. Thus, a single bit is required to reconstruct the second of the two di- 


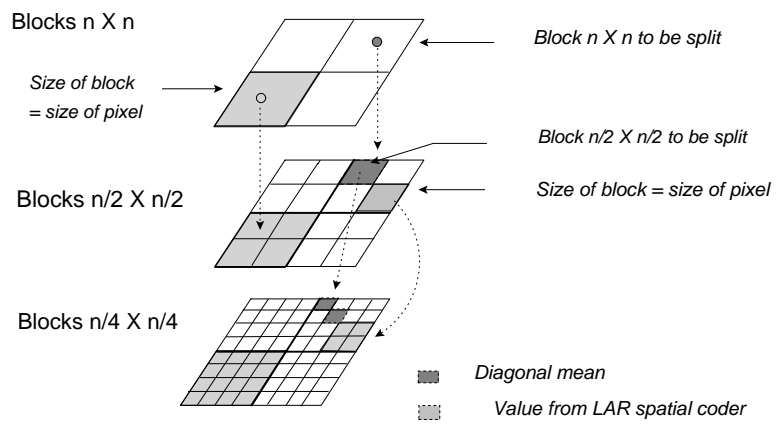

Fig. 4. Block values to be predicted

agonal values, i.e. 0.25 average bit per block and per level. Applying a minimal quantization removed this additional bit and the number of coded symbols is exactly equal to the number of blocks of our LAR representation.

Note that this pyramidal decomposition modify the initial LAR representation where each block value is the average of all pixels within this block. Nevertheless, a slight difference is introduced. Indeed, except from the $2 \times 2$ blocks, all the blocks correspond to flat areas (by construction), and the average luminance of whole pixels is quite similar to the diagonal pixels average (Fig. 5).

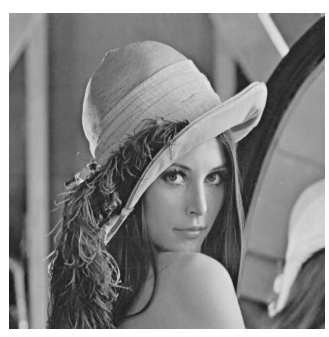

(a)

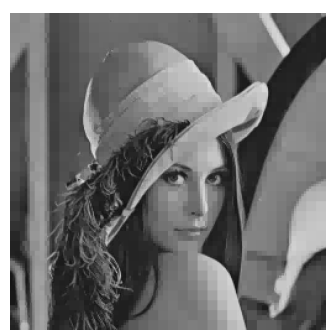

(c)

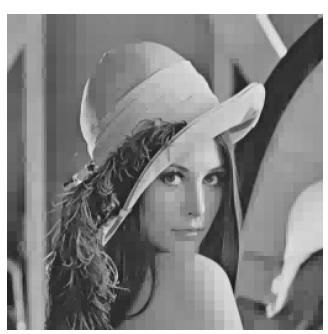

(b)

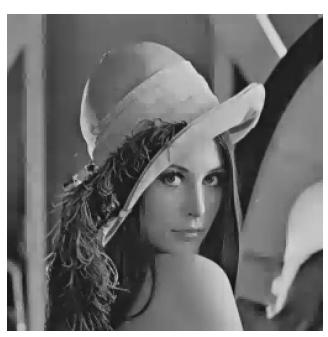

(d)
Fig. 5. (a) Original image. (b) Low resolution initial LAR image. (c) Low resolution new LAR image. (d) Post-processed new LAR image

\subsection{Experimental results}

The table 1 gives the entropies resulting from the pyramidal approach compared to the ones obtained by the original scheme. One can observe that the entropy is equivalent for both techniques, but now the encoding scheme is scalable.

\begin{tabular}{|c|c|c|c|c|}
\hline & \multicolumn{2}{|c|}{$\begin{array}{c}\text { Entropy (bit per } \\
\text { block) }\end{array}$} & \multicolumn{2}{c|}{$\begin{array}{c}\text { Global entropy } \\
\text { (bpp) }\end{array}$} \\
\hline Image name & Initial & New & Initial & New \\
\hline Baboon & 6.73 & 6.79 & 1.05 & 1.06 \\
\hline Barb & 6.45 & 6.54 & 0.67 & 0.68 \\
\hline Bike & 6.68 & 7.17 & 0.41 & 0.44 \\
\hline Cafe & 6.80 & 7.12 & 0.84 & 0.88 \\
\hline Finger & 6.51 & 6.46 & 1.36 & 1.35 \\
\hline Hotely & 6.25 & 6.61 & 0.52 & 0.55 \\
\hline Lena & 6.17 & 5.98 & 0.32 & 0.33 \\
\hline Zelda & 5.81 & 6.15 & 0.17 & 0.18 \\
\hline
\end{tabular}

Table 1. Compression results for low resolution image (LAR coder) : original and new method.

\section{SCALABLE LOSSLESS CODING}

The previous stage provides a low resolution image. It also separates two types of blocks :

- flat areas in all $N \times N$ blocks with $N \in\{4,8,16\}$, characterized by a low entropy,

- contour areas in $2 \times 2$ blocks, characterized by an high entropy.

The extension of the method to a lossless scheme consists in coding the error image (texture) which results from the LAR compressed image and the original one. Each $N \times N$ block in the LAR representation is decomposed until full resolution according to our pyramidal decomposition principle. Next sub-section deals more particulary with the separation of the entropy into different laws.

\subsection{Entropy laws and implicit context modeling}

Global entropy can be reduced when different classes of symbols following the same law can be isolated. This principle is often used in lossless coding methods through the context modeling technique [1] : a local estimation of a pixel activity leads to its classification into one a priori law.

Our method provides a straightforward separation of the laws at two different levels :

- from the block size : $2 \times 2$ blocks are located on strong edges in the image inducing an high average entropy error, whereas other blocks contain local homogenous texture,

- from the several passes of each decomposition level : the bit resulting from the diagonal mean is transmitted separately. Values from passes 2 and 3 significantly differ.

The table 2 gives an example of the final entropy, when separating the LAR representation, texture and passes 2 and 
3 error values. The last level (full resolution) distinguishes the $2 \times 2$ blocks from the others. This divided bitstream gives much more interesting results than a global coding (Tab. 3). Actually, an implicit context modeling is made, due the fact that the quad-tree decomposition is already content based.

\begin{tabular}{|c|l|c|c|}
\hline \multirow{2}{*}{ Level } & \multirow{2}{*}{ Size of blocks } & \multicolumn{2}{|c|}{ Entropy $(\mathrm{bpb})$} \\
\cline { 2 - 4 } & & Pass 2 & Pass 3 \\
\hline \multirow{2}{*}{3} & Texture : $16 \times 16$ & 4.25 & 3.93 \\
\cline { 2 - 4 } & LAR : $8 \times 8,4 \times 4,2 \times 2$ & 5.79 & 5.92 \\
\hline \multirow{2}{*}{2} & Texture : $16 \times 16,8 \times 8$ & 3.93 & 3.97 \\
\cline { 2 - 4 } & LAR : $\times 4,2 \times 2$ & 5.74 & 6.06 \\
\hline \multirow{2}{*}{1} & Texture : $16 \times 16,8 \times 8,4 \times 4$ & 3.72 & 4.06 \\
\cline { 2 - 4 } & LAR : $\times 2$ & 5.82 & 6.09 \\
\hline \multirow{2}{*}{0} & Texture : $16 \times 16,8 \times 8,4 \times 4$ & 3.54 & 3.92 \\
\cline { 2 - 4 } & Edges : $2 \times 2$ & 5.12 & 5.34 \\
\hline \hline 4 & All blocks & \multicolumn{2}{|c|}{ Pass 1:6.07 } \\
\hline
\end{tabular}

Table 2. Lossless coding of Lena image.

The table 3 presents also the result of the application of the CALIC algorithm on the images. One can see that this the state-of-the-art method is a bit better than our compression scheme, but our pyramidal approach has the advantage to be spatially scalable.

\begin{tabular}{|c|c|c|c|}
\hline & \multicolumn{3}{|c|}{ Entropy (bpp) } \\
\hline Image & $\begin{array}{c}\text { Proposed } \\
(1 \text { law })\end{array}$ & $\begin{array}{c}\text { Proposed } \\
\text { (sev. laws) }\end{array}$ & CALIC \\
\hline Baboon & 7.02 & 6.09 & 6.14 \\
\hline Barb & 5.88 & 5.02 & 4.93 \\
\hline Bike & 5.40 & 4.63 & 4.53 \\
\hline Cafe & 6.41 & 5.50 & 5.37 \\
\hline Finger & 6.56 & 5.75 & 5.52 \\
\hline Hotely & 5.66 & 4.89 & 4.56 \\
\hline Lena & 5.07 & 4.40 & 4.33 \\
\hline Zelda & 4.80 & 4.21 & 3.98 \\
\hline Average & 5.85 & 5.06 & 4.92 \\
\hline
\end{tabular}

Table 3. Compression results for lossless coding of images.

\subsection{Influence of the $L A R$ parameter}

The threshold of the morphological gradient is the unique parameter. It determines the quantity of information respectively allocated to low resolution image and texture. The figure 6 gives the entropy of Lena image when this threshold varies. One can see that the dispersion of the resulting values is small for a given range of thresholds. Thus, the method is not very sensitive to this parameter : it can then be a priori fixed.

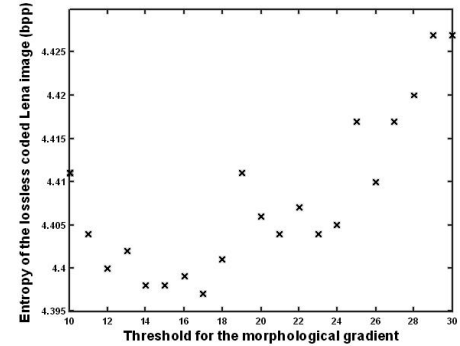

Fig. 6. Influence of the gradient threshold.

\section{CONCLUSION AND PERSPECTIVES}

This paper has presented an original technique for scalable lossy and lossless compression. The knowledge of the quadtree data structure is sufficient to improve significantly the compression ratio for the lossless coding, by gathering and coding separately the information of the same nature.

By means of an unique algorithm, one can choose either a lossy or a lossless image coding. The configuration of the codec is simplified since the choice of the gradient estimation parameter has no significant impact on the bit rate. Moreover, the properties of the previous LAR coder are kept. The scalability here introduced in the scheme extend the possibilities of the codec.

Future research will be made in the adaptation of the prediction according to the nature of the information (contours or texture).

\section{REFERENCES}

[1] X. Wu, N. Memon, and K. Sayood, "A context-based, adaptive, lossless/nearly-lossless coding scheme for continuoustone images," in ISO/IEC JTC 1/SC 29/WG 1, vol. 202, July 1995.

[2] A. Said and W. Pearlman, "Reversible image compression via multiresolution representation and predictive coding," in $\mathrm{Vi}$ sual Communication and Image Processing. SPIE, 1993, vol. 269.

[3] B. Meyer and P. Tisher, "TMW : a new method for lossless image compression," Proc. Picture Coding Symposium, Berlin, 1077.

[4] O. Deforges and J. Ronsin, "Region of interest coding for low bit-rate image transmission," ICME'00, 2000.

[5] C. Chen, "Adaptive transform coding via quad-tree based variable block-size DCT," ICASSP'89, pp. 1854-1856, 1989.

[6] X. Wu, "Lossless Compression of Continuous-Tone Images, via Context Selection, Quantization and Modelling," IEEE Trans. on Image Processing, vol. 6, no. 5, pp. 656-664, 1996. 